\title{
UN CASCO DE TIPO MONTEFORTINO LOCALIZADO EN LA PARTIDA DE LA CARROVA (AMPOSTA, MONTSIÀ, TARRAGONA)
}

POR

\author{
DAVID GARCÍA i RUBERT
}

\section{RESUMEN - ABSTRACT}

Pretendemos con esta breve nota dar a conocer la existencia de tres fragmentos de bronce pertenecientes a un nuevo ejemplar de casco Montefortino. El contexto de su localización y sus características decorativas lo acercan a los individuos recuperados en las necrópolis del sudeste peninsular, distanciándolo un tanto de los ejemplares catalanes conocidos hasta el momento.

The aim of this note is to present three bronze fragments from a new Montefortino helmet. From its localisation context and decorative characteristics, we maintain a relation between this helmet and others found in the southeastern Iberian cemeteries, placing this helmet far from the catalonian examples.

\section{PALABRAS CLAVE - KEY WORDS}

Casco Montefortino. Ibérico Tardío. Ustrinum. Ajuar funerario. Necrópolis de incineración.

Montefortino helmet. Late Iberian. Ustrinum. Grave goods. Incineration cemetery.

\section{INTRODUCCIÓN}

El objetivo de este artículo es dar a conocer la existencia de un nuevo casco de tipo Montefortino en Catalunya. Como resultado de los trabajos de prospección que habían de conducir a la realización de nuestra Tesis de Licenciatura, centrada en el poblamiento de época protohistórica en la comarca tarraconense del Montsià ${ }^{1}$, tuvimos la ocasión de estudiar un conjunto de piezas de metal que se encontraban en manos de un particular. Este lote de elementos, que dejando a un lado los fragmentos pertenecientes al casco incluía una pequeña asa de bronce con una decoración en forma de cabeza de barbudo, una moneda, y un par de pequeñas campanas de bronce, formaba parte de un conjunto mayor en el que cabe señalar la presencia de varias jarritas bitroncocónicas de cerámica gris de la costa catalana, diversas fusayolas, un kalathos con decoración pintada de tipo floral, estilizada, de color rojo vinoso y un oinochoe con decoración similar a la de la pieza anterior ${ }^{2}$.

1 GARCIA i RUBERT, D. (1999): Evolució del poblament a la comarca del Montsià (ss. VII a. C.- III d. C.), tesis de licenciatura inédita, Universitat de Barcelona.

2 El conjunto cerámico se encuentra actualmente depositado en el Museu Comarcal del Montsià, pendiente de un estudio pormenorizado de sus características generales. 
Los materiales proceden de una localización fortuita, realizada por un aficionado ampostino, de un enterramiento, aparentemente único, situado a media pendiente de la pequeña elevación de La Coma, en la partida de La Carrova, en la orilla derecha del río Ebro (fig. 1). Las informaciones orales indican que el conjunto de elementos muebles localizado se encontraba en el interior de una gran depresión de forma pseudocircular que en ese punto formaba la roca madre (piedra caliza local). Avisados por parte de la conservadora del Museu Comarcal del Montsià, Sra. M Mar Villalbí, de la localización de estos elementos, pudimos constatar in situ como el citado lugar de enterramiento presentaba claras evidencias de la realización de un intenso fuego en su interior, hasta el punto de que la roca había sufrido un proceso de rubefacción en diversos lugares. Este hecho permitía explicar a la perfección las evidentes muestras de cremación que presentaban la mayoría de las piezas recuperadas, y de la misma forma permite clasificar tipológicamente el sitio como un probable ustrinum. De igual forma, la localización de diversos fragmentos cerámicos en las proximidades nos permitió corroborar la veracidad de la noticia de la localización en ese lugar del conjunto metálico y cerámico objeto de este comentario.

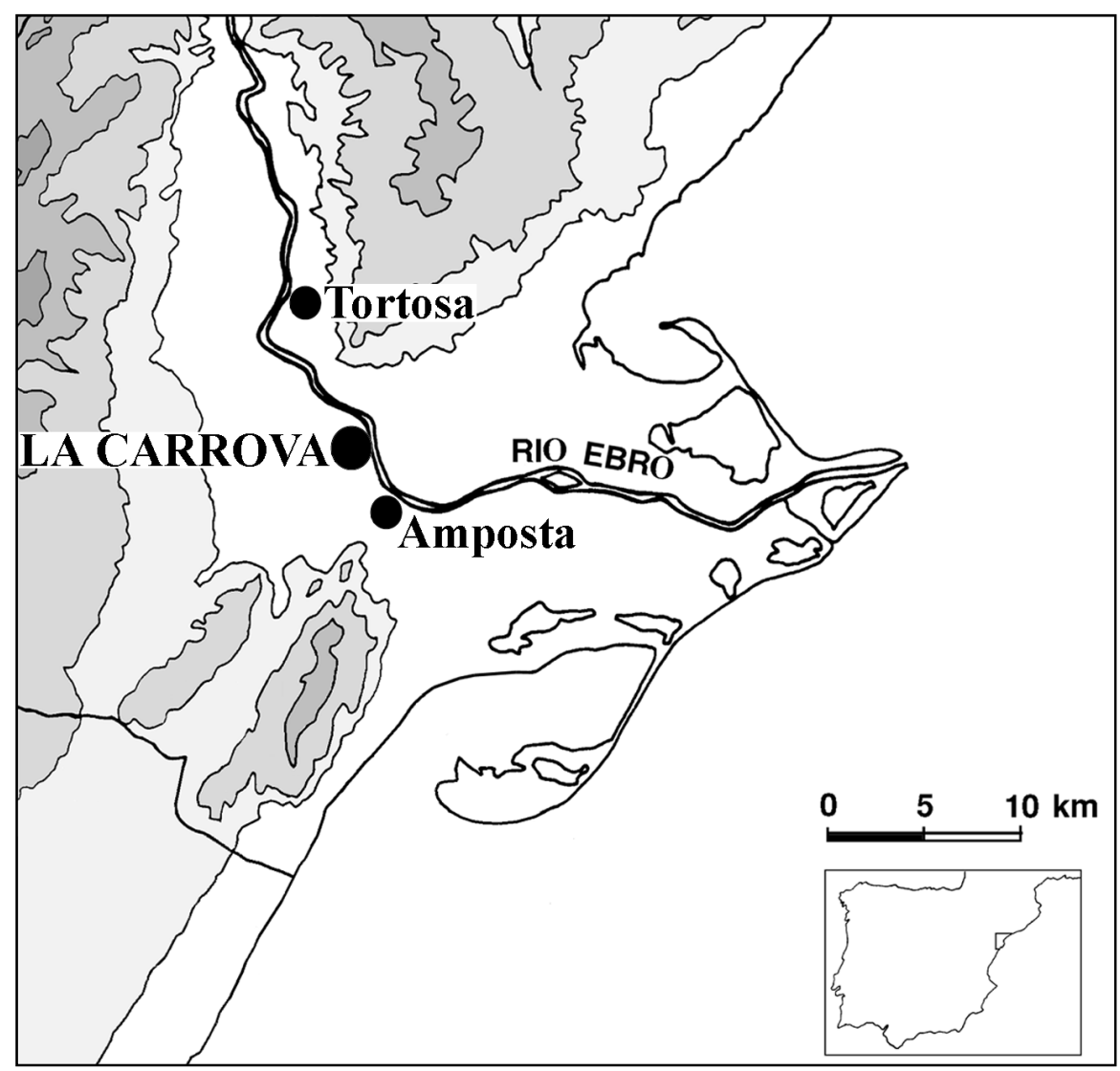

Fig. 1. Mapa de situación de La Carrova.

Del conjunto metálico destacamos en esta breve nota los tres fragmentos de bronce pertenecientes a un casco de tipo Montefortino, o «gorra de jockey» (fig. 3), que son estudiados a continuación. 


\section{DESCRIPCIÓN DE LOS FRAGMENTOS}

Primer fragmento (fig. 2, a): El primer fragmento pertenece a la parte superior del casco. Conserva el botón terminal, de forma ovoidal, truncado en su parte superior a causa de la existencia del orificio de acople de la crinera. El orificio no atraviesa todo el botón, ya que este presenta una estructura general maciza. El botón presenta, además, una decoración formada por una doble hilera de ovas incisas, separadas por diversas líneas paralelas también incisas. Altura del botón desde su base: $25 \mathrm{~mm}$. Diámetro máximo del botón: $26 \mathrm{~mm}$.

Segundo fragmento (fig. 2, b): El segundo fragmento pertenece al tercio inferior del casco. Muestra una decoración seriada horizontal que, de arriba a abajo, queda estructurada por dos grupos de tres líneas incisas paralelas, otro grupo formado por una línea incisa y una banda con decoración de espiga o de un fino zigzag, y un reborde amplio decorado con líneas incisas paralelas, dispuestas oblicuamente y a distancias equidistantes, tradicionalmente conocido como decoración de cableado. Conserva media bisagra, unida al cuerpo principal del casco mediante un par de remaches; con toda seguridad, este último elemento se tendría que poner en relación con algún elemento similar, no localizado, situado en conexión con la paragnatide. Anchura de la pared del casco: 1,6 mm.

Tercer fragmento (fig. 2, c): El tercer fragmento, que con toda seguridad ha de pertenecer a una de las paragnatides, elemento que raramente se conserva en los ejemplares de cascos recuperados en la Península Ibérica (Quesada, 1992: 65), presenta una decoración formada por tres acanaladuras finas paralelas, las cuales insinúan un dibujo curvo. Dispone asimismo de un par de orificios, utilizados para adaptar en ellos el barboquejo. Anchura: 1,5 mm.

La decoración es en conjunto de muy buena calidad, realizada con mucho esmero y homogeneidad. En cambio, el estado de conservación de la pieza es bastante deficiente como consecuencia principalmente del contacto directo con el fuego, del cual presenta múltiples evidencias.

\section{ANÁLISIS}

El tipo de decoración que documentamos en el botón terminal es bastante habitual, pudiendo presentarse como paralelos los ejemplares de Benicarló (Castelló) (Oliver, 1987/88), Tumba 146 del Cabecico del Tesoro (Verdolay, Múrcia) (Quesada, 1989: 18-19) o Pago de Gorrita (Valladolid). La decoración del reborde en la parte inferior del casco también es muy frecuente, documentándose en los ejemplos de los cascos de Aljezur (Varcea de Misericòrdia, Algarve, Portugal), Tumba 428 del Cabecico del Tesoro (Verdolay, Murcia), Caldelos de Tuy (Pontevedra), Tumbas 1, 2, 3 i 4 de Galera (Granada), Lacimurga (Navalvillar de Pela-Puebla de Alcocer, Badajoz) (Aguilar, Guichard, 1993: 36), Tumbas 7, 31 i 110 de la necrópolis de Les Corts (Empúries, Girona), Ses Paises (Artà, Mallorca), y Villaricos-C (Almería). Por último, la combinación de los dos tipos de decoración la podemos encontrar, por ejemplo, en los ejemplares de Alarcos (Ciudad Real), Pozo Moro (Chinchilla, Albacete), Almaciles (Granada) (Quesada, 1992) y muy especialmente en el de la tumba O de la Hoya de Santa Ana (Albacete) (Blánquez, 1990a: 275-291). García-Mauriño y F. Quesada han ido recopilando la mayoría de estos cascos peninsulares (Quesada, 1992) (GarcíaMauriño, 1993). Con todo, el casco de la Hoya de Santa resulta el más similar al ejemplar de La Carrova.

García Mauriño clasifica este tipo de casco como tipo I (forma general semiesférica, guardanucas inclinado, botón troncocónico macizo y perforado preferentemente, dos remaches para la bisagra, decoración generalizada y reborde inferior con decoración cableada). El que es objeto de estudio en este trabajo tendría que encuadrarse específicamente en el subti- 

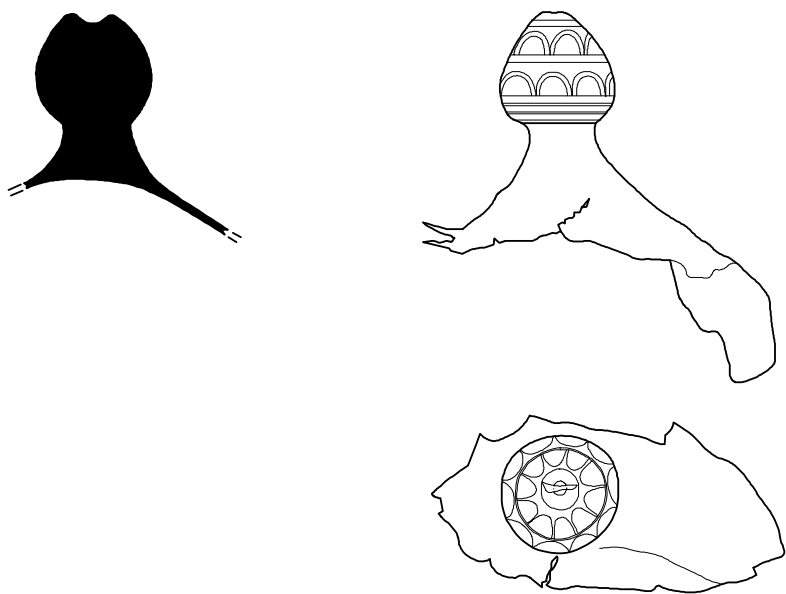

a
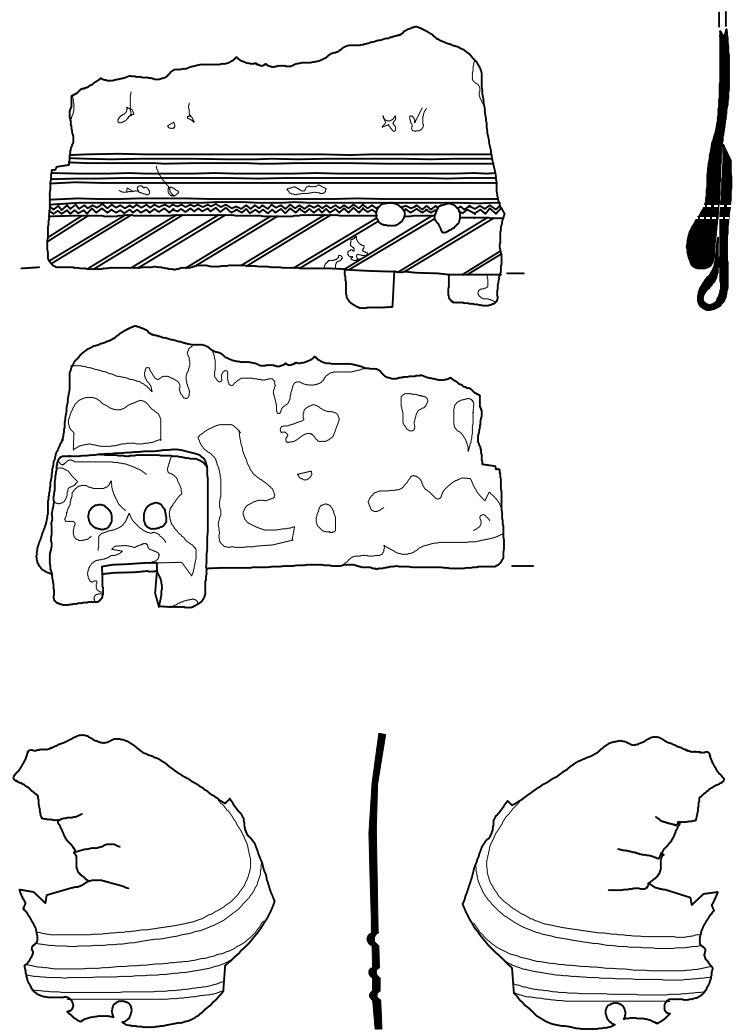

C

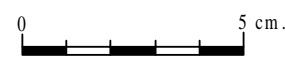

Fig. 2. Fragmentos del casco de tipo Montefortino. 
po Ia, ya que, como aquellos, el botón presenta una decoración de ovas, y la decoración de la parte inferior del casco está formada por una línea de espiga o en zigzag. La cronología que este autor propone para el tipo I es de finales del siglo III-siglo II a. C. (García-Mauriño, 1993), coincidiendo con la cronología propuesta por otros autores (Quesada, 1989: 19). Otras clasificaciones lo englobarían en el tipo B de Robinson (Robinson, 1975) o en el B de Abásolo y Pérez (Abásolo, Pérez, 1980) (Abásolo, Pérez 1985).

En el conjunto de la península Ibérica existen, como ya hemos podido observar, diversos cascos de tipo Montefortino que presentan una decoración del botón terminal o remate superior similar o prácticamente idéntico al de La Carrova ${ }^{3}$. F. Quesada, por ejemplo, documenta uno muy similar en la sepultura 146 de la necrópolis del Cabecico del Tesoro (Murcia), datado, por su asociación con un guttus campaniense, forma Morel 8151/Lamb. 46, hacia finales del siglo III o principios del II a. C. (Quesada, 1989: 18-19, lám. IV). Mucho más cercanos a la comarca del Montsià son los tres cascos pertenecientes a la misma tipología recuperados de forma fortuita bajo las aguas de la costa de Benicarló (Castelló), en las proximidades de un antiguo fondeadero, actualmente cubierto por el mar. Uno de ellos presenta la misma decoración de dos líneas paralelas de ovas incisas en el remate superior macizo, y tomando como base este motivo el autor propone una clasificación tipológica dentro de la categorización Monterfortino B, piezas más evolucionadas dentro de la gran familia que forman estos cascos. La cronología propuesta se sitúa entre los siglos III y I a. C., en base al material cerámico anfórico recuperado en el mismo sector del fondeadero (Oliver, 1987/88: 207).

La mayoría de los cascos de tipo Montefortino localizados en la península Ibérica han sido encontrados en contextos de necrópolis, y estas prácticamente son siempre de incineración (García-Mauriño, 1993). Cuando aparecen en este tipo de contextos, normalmente se encuentran fragmentados o presentan fuertes golpes, ya sea debido a la acción del fuego o a una intencionalidad destructiva consecuencia del ritual de enterramiento utilizado (Quesada, 1989: 17). El ejemplar de La Carrova presenta suficientes muestras de destrucción como para poder afirmar que durante el proceso ritual fue objeto de un trato similar al anteriormente descrito y constatado en otras necrópolis.

El origen de este tipo de piezas se había buscado tradicionalmente en un ambiente céltico, del norte de Europa (Blázquez, 1959-60) (Stary, 1982). Con todo, los últimos trabajos proponen un origen itálico, y en época romana, para a la mayoría de cascos de la Península, excepto en el caso de los ejemplares catalanes de La Pedrera (Vallfogona de Balaguer, Lleida), Can Miralles (Barcelona) y Les Corts (Empúries, Girona), de características acusadamente célticas tanto desde el punto de vista formal como, a veces, por el contexto. Los restantes cascos peninsulares han sido localizados en contextos claramente ibéricos, concentrados principalmente en el sudeste y, en menor medida, en el Levante (Quesada, 1992: 68-71).

Concluyendo, consideramos que la cronología de este casco ha de cifrarse a finales del siglo III a. C. o principios de la siguiente centuria ${ }^{4}$, hecho que por otra parte viene a coincidir bastante con la datación de la mayoría de cascos de este tipo presentes en la Península Ibérica, (García-Mauriño, 1986) (García-Mauriño, 1993) (Quesada, 1992). También coincide esta datación con la que, por ahora, proporciona una primera aproximación a los elementos muebles cerámicos recuperados ${ }^{5}$. En relación a este último hecho, cabe decir que el tipo de

3 Como curiosidad, destacamos el casco Montefortino que durante años formó parte de la colección de referencia del Instituto de Arqueología de la Universitat de Barcelona. Se desconocía totalmente su procedencia, aunque con todo fue atribuido a la necrópolis de la Galera (Granada), sin mucho fundamento. Se trataba de un casco que presentaba evidentes muestras de haber estado en contacto con un fuego intenso, y posteriormente doblado sobre si mismo. Cuando fue restaurado, fue posible determinar su tipología. La decoración de líneas paralelas oblicuas que presenta en su parte terminal inferior lo acercan también al casco documentado en La Carrova (Maluquer de Motes, 1987).

4 Agradecemos las apreciaciones que sobre este particular nos ha proporcionado el Dr. Fernando Quesada.

5 Queremos desde aquí agradecer a la Dra. Ma . J. Conde las apreciaciones realizadas en relación a la tipología decorativa y formal de estos elementos cerámicos. 


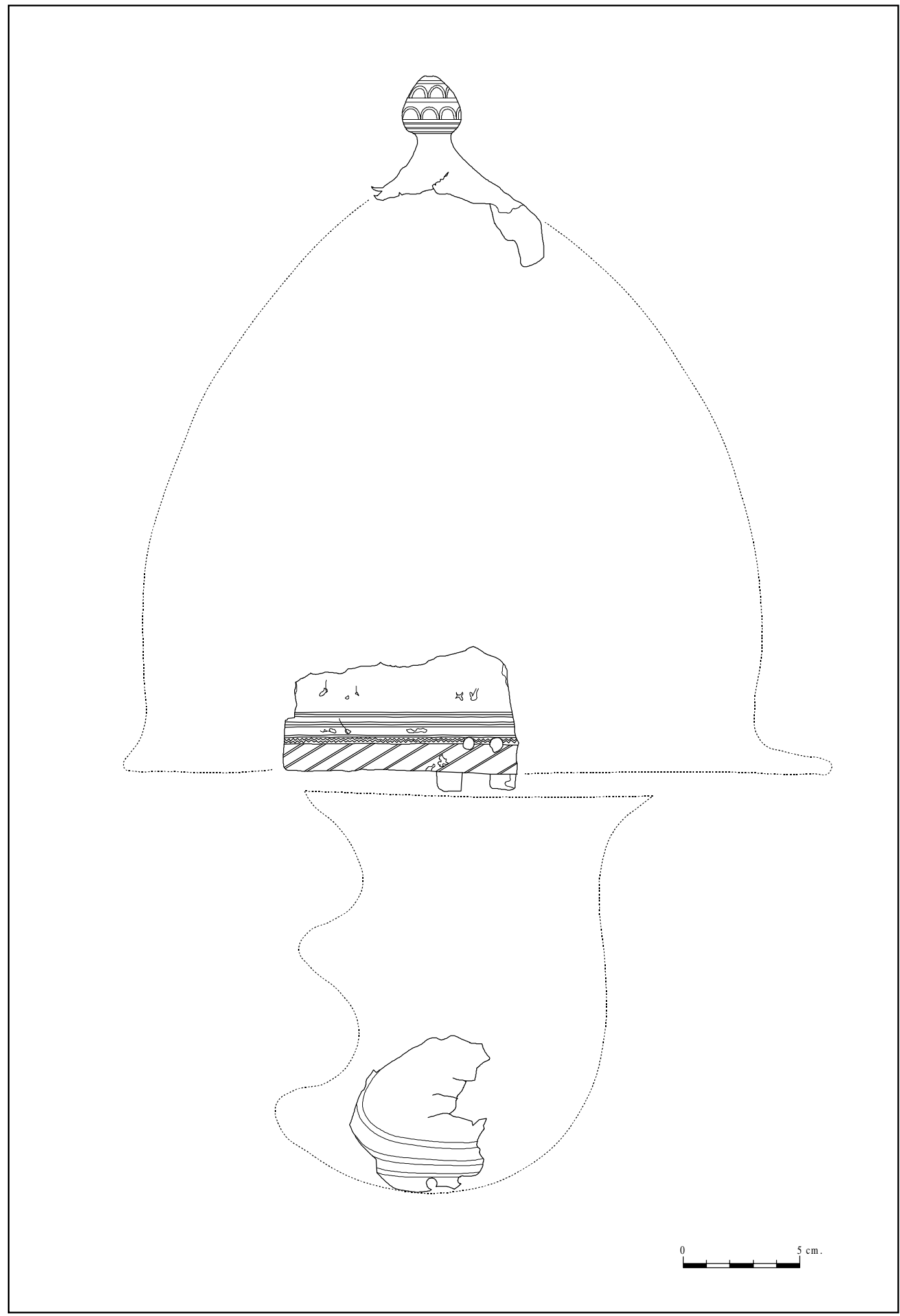

Fig. 3. Reconstrucción ideal del casco. 
ajuar funerario documentado (asociación kalathos-oinochoe, tipo de decoración pintada de estas piezas, presencia de un buen número de fusayolas, presencia de un casco de tipo Montefortino) no presenta paralelos en el área, y para encontrarlos tenemos que situarnos en el sudeste peninsular. Como ejemplo podemos nombrar las similitudes con algunos conjuntos de la necrópolis de la Hoya de Santa Ana (Chinchilla, Albacete) (Blánquez, 1990b) o del Cabecico del Tesoro (Verdolay, Murcia) (Quesada, Sánchez Meseguer, 1990), entre otros. Esta interesante problemática, que ha sido tratada por nosotros en un trabajo reciente inédito (Garcia i Rubert, 1999: 74-79), será objeto próximamente de un estudio en profundidad. Se puede afirmar, en todo caso, que su contexto es claramente ibérico, por el tipo de ajuar y el ritual documentado. Desconocemos, evidentemente, su posible relación con el conflicto de la Segunda Guerra Púnica, aunque la posible atribución de su uso a agentes púnicos y mercenarios ibéricos al servicio de Cartago durante este conflicto, tesis propuesta por F. Quesada (Quesada, 1995: 168) (Quesada, 1992: 71), así como el referente que para este enterramiento suponen las necrópolis del sudeste peninsular, abre las puertas a la posibilidad de establecer esta conexión.

\author{
DAVID GARCÍA I RUBERT \\ Becario FPI del MEC. Departament de Prehistòria, Història Antiga i Arqueologia \\ Facultat de Geografia i Història, Universitat de Barcelona. \\ C/Baldiri i Reixac, s/n, Barcelona 08028. E-mail: dgarcia@trivium.gh.ub.es
}

\title{
BIBLIOGRAFÍA
}

ABÁsolo, J.A.; Pérez RodríGuez, F. (1980): El casco céltico de Gorrita (Valladolid), Boletín del Seminario de Estudios de Arte y Arqueología, XLVI: 93-119.

ABÁsolo, J.A.; PÉREZ RodríGUeZ, F. (1985): El casco celtoitálico de Gorrita (Valladolid) y sus paralelos europeos, Comunicaciones del Primer Congreso Internacional de Historia Militar, vol. II, Zaragoza: 41-55.

Aguilar, A; Guichard, P. (1993): Lacimurga. La ciudad antigua y su entorno, Revista de Arqueología, nº 144: 32-38.

BLÁNQUEZ PÉREZ, J. (1990a): La formación del mundo ibérico en el sureste de la Meseta. (Estudio arqueológico de las necrópolis ibéricas de la provincia de Albacete), Instituto de Estudios Albacetenses, CSIC, Albacete, 658 pp.

BLÁNQUeZ PÉREZ, J. (1990b): Las necrópolis ibéricas en el sudeste de la Meseta, Congreso de Arqueología ibérica: las necrópolis, Serie Varia, 1, Madrid: 235-278.

BLÁZqueZ, J. M. (1959-69): Cascos celtas inéditos. Notas sobre los cascos hispánicos, Boletín de la Comisión de Monumentos de Orense, XX: 371-387.

García Mauriño, J. (1986) : Los cascos de La Tène en la Península Ibérica. Memoria de licenciatura inédita, U. Complutense de Madrid.

García Mauriño, J. (1993): Los cascos de tipo Montefortino en la Península Ibérica, Complutum, 4: 95-146.

GARCIA i Rubert, D. (1999): Evolució del poblament a la comarca del Montsià (ss. VII a.C.- III d. C.). Tesis de licenciatura inédita, Universitat de Barcelona.

MALUQueR De Motes, J. (1987): Un casco ibérico probablemente de la necrópolis de Galera (Granada) en el Instituto de Arqueología de la Universidad de Barcelona, Archivo de Prehistoria Levantina, Homenaje a D. Domingo Fletcher, Tomo I, Vol. XVII, Valencia: 257-260.

Oliver FoIX, A. (1987/88): Tres yelmos tipo Montefortino hallados en Benicarló (Castellón), Cuadernos de Prehistoria y Arqueología Castellonenses, 13, Castelló: 205-212. 
QUESADA SANZ, F. (1989): Armamento, guerra y sociedad en la necrópolis ibérica de El Cabecico del Tesoro (Murcia, España), 2 volúmenes, BAR International Serie, 502(ii).

Quesada SANZ, F. (1992): El casco de Almaciles (Granada) y la cuestión de los cascos de tipo «Montefortino» en la Península Ibérica, Verdolay, 4: 65-73.

QUESADA SANZ, F. (1995): Las armas en la sociedad ibérica: 10 preguntas fundamentales, El mundo ibérico: una nueva imagen en los albores del año 2000, Catalogo de la exposición, Junta de comunidades de Castilla-La Mancha: 159-169.

Quesada SAnZ, F.; SÁNCHEZ MeSEGuER, J.L. (1990): La necrópolis ibérica del Cabecico del Tesoro (Verdolay, Murcia), Congreso de Arqueología ibérica: las necrópolis, Serie Varia, 1, Madrid: 349-396.

ROBINSON, H. R. (1975): The armour of imperial Rome, Londres.

STARY, P. (1982): Keltische waffen auf der Iberischen Halbinsel, Madrider Mitteilungen, 23: 114-144. 